\title{
Injuries and the risk of disability in teenagers and young adults
}

\author{
Maggie Barker, Chris Power, Ian Roberts
}

\begin{abstract}
Objective-To examine the risk of disability from unintentional injury in teenagers and young adults.

Methods - Analyses of data from the National Child Development Study, a follow up study of $98 \%$ of all children born in England, Scotland, and Wales in one week in March, 1958. In 1981, 12537 study participants, $76 \%$ of the original cohort, were asked about unintentional injuries since age 16 years requiring hospital treatment, and whether these injuries resulted in permanent disability.

Results $-62 \%$ of men and $26 \%$ of women reported at least one accident since age 16 resulting in injury that required hospital treatment. Of these accidents, $3.2 \%$ caused permanent disability. The risk of disability increased with accident frequency. Injuries requiring hospital admission carried the highest risk of disability (9.7\%). However, $54 \%$ of permanent disability reported by men and $74 \%$ reported by women resulted from injuries treated as outpatients. Road traffic accidents caused $42 \%$ of admissions and $31 \%$ of disability. Fractures constituted $21 \%$ of all injuries but were responsible for $32 \%$ of permanent disabilities. Of the permanent disabilities resulting from work related accidents, $82 \%$ involved the hand. Of the permanent disabilities resulting from accidents in the home, $32 \%$ involved the hand.
\end{abstract}

Conclusions - The targeting of prevention strategies towards the major causes of injury mortality may have a smaller impact on population levels of injury related disability. Non-life threatening injuries, in particular injuries to the hand and limb fractures, resulting from accidents in the workplace, the home, and during sports, make a significant contribution to the prevalence of permanent injury related disability in young adults.

(Arch Dis Child 1996;75:156-158)

Keywords: injuries, disability, young adults, cohort.

Accidental injury is a leading cause of death in children and young adults in the United Kingdom, and an important cause of acquired disability. ${ }^{1}$ In 1990 , the government strategy for health, The Health of the Nation, established the reduction of ill health, disability, and death from accidents as a national priority. ${ }^{2}$ Although the white paper clearly acknowledged the importance of injury related disability, a lack of information on its determinants remains an impediment to progress in this area. ${ }^{3}$ While it may be appropriate to assume that the major causes of injury mortality are also the major contributors to injury related disability, there are some important exceptions. For example, most life threatening visceral injuries leave little or no disability. In contrast, isolated lower limb injuries are rarely life threatening but may be associated with prolonged periods of disability and handicap. ${ }^{4}$ We used data from the National Child Development Study to assess the risk of disability associated with different types of unintentional injury.

\section{Methods}

The National Child Development Study is a follow up study of $98 \%$ of all children born in England, Scotland and Wales in one week in March, 1958. The study methodology has been described in detail elsewhere. ${ }^{5}$ To date there have been five follow up contacts, at ages $7,11,16,23$, and 33 . Immigrants born in the relevant week were included in the sweeps at ages 7,11 , and 16 . The current analyses are based on information collected in the 1981 sweep of 12537 cohort members, at age 23 years. Seventy six per cent of all study members still alive and resident in Britain in 1981 were followed up.

At the 1981 follow up, information was collected on accidents occurring between the ages of 16 and 23 years that required hospital admission, accident and emergency attendance, or outpatient department attendance. For the first eight recorded accidents information was collected on: age at time of accident, type of accident, hospital or outpatient attendance, and injury description. Injury related disability was ascertained by the question 'Has this (or have any of these) accident(s) resulted in any permanent disability?' Those reporting a disability were asked to provide a brief description of the nature of the disability. In situations where subjects reported disability and more than one accident, the injury description was compared with the disability description to determine which accident had caused the disability. Similarly, in situations where two disabilities were mentioned, comparisons were made to determine whether they were caused by the same or separate injuries.

The risk of permanent disability following an accident was estimated from the total number of accidents reported. Relative risk (RR)and 95\% confidence interval (CI) were calculated HA1 3EX. 
Table 1 Risk of injury related disablility and accident frequency

\begin{tabular}{lllll}
\hline Accidents & Subjects & Male \% & Female \% & $R R(95 \%$ CI) \\
\hline One & 2901 & 59 & 41 & 1.00 \\
Two & 1243 & 76 & 24 & $1.30(0.99$ to 1.71$)$ \\
Three & 628 & 82 & 18 & $2.16(1.61$ to 3.28$)$ \\
Four & 327 & 90 & 10 & $2.30(1.61$ to 3.28$)$ \\
Five or more & 367 & 89 & 11 & $2.34(1.67$ to 3.28$)$ \\
\hline
\end{tabular}

$\mathrm{RR}=$ relative risk; $\mathrm{CI}=$ confidence interval

Table 2 Accidents presenting for hospital treatment and percentage resulting in admission

\begin{tabular}{lcccc}
\hline Accident & Male (\%) & Female (\%) & Total (\%) & Admissions* (\%) \\
\hline Road pedestrian & $138(2)$ & $91(4)$ & $229(2)$ & 32.0 \\
Road other & $1431(17)$ & $443(18)$ & $1874(17)$ & 28.0 \\
Work & $2821(33)$ & $484(20)$ & $3305(30)$ & 6.1 \\
Home & $721(9)$ & $602(25)$ & $1323(12)$ & 6.7 \\
Sports & $2212(26)$ & $308(13)$ & $2520(23)$ & 11.5 \\
Other & $1207(14)$ & $478(20)$ & $1685(15)$ & 14.3 \\
All & $8530(100)$ & $2406(100)$ & $10936(100)$ & 13.0 \\
\hline
\end{tabular}

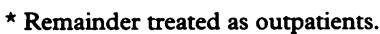

Table 3 Accidents resulting in permanent disability

\begin{tabular}{lccrrrrr}
\hline & \multicolumn{3}{l}{ Inpatient } & & \multicolumn{2}{c}{ Outpatient } & \\
\cline { 2 - 3 } Accident & Male (\%) & Female (\%) & & Male (\%) & Female (\%) & Total (\%) \\
\hline Road pedestrian & $49(12.2)$ & $24(12.5)$ & & $88(1.1)$ & $67(7.5)$ & $228(6.6)$ \\
Road other & $403(12.2)$ & $118(8.5)$ & & $1019(2.2)$ & $319(5.0)$ & $1859(5.2)$ \\
Work & $172(16.9)$ & $27(22.2)$ & & $2621(1.3)$ & $453(2.9)$ & $3273(2.5)$ \\
Home & $38(5.3)$ & $50(2.0)$ & & $673(2.7)$ & $546(2.4)$ & $1307(2.6)$ \\
Sports & $255(6.3)$ & $32(6.3)$ & & $1936(1.9)$ & $272(4.4)$ & $2495(2.6)$ \\
Other & $178(2.8)$ & $59(8.5)$ & & $1012(1.7)$ & $411(4.1)$ & $1660(2.6)$ \\
All & $1095(9.9)$ & $310(9.0)$ & & $7349(1.9)$ & $2068(3.8)$ & $10822(3.2)$ \\
\hline
\end{tabular}

for subjects reporting more than one accident compared with those reporting only one.

\section{Results}

A total of 11187 accidents were reported by 5512 subjects. Sixty two per cent of men and $26 \%$ of women reported at least one accident requiring hospital admission, accident and emergency attendance, or hospital outpatient attendance since their 16th birthday. Men were more likely to report more than one accident, accounting for $93 \%$ of those reporting five or more accidents. A total of 358 permanent disabilities were reported by 349 subjects. Of the 11187 accidents, $3.2 \%$ resulted in permanent disabilities. The proportion of accidents resulting in permanent disability was significantly higher $(p<0.01)$ for women $(4.4 \%)$ than for men $(2.9 \%)$. The risk of permanent disability was strongly associated with the number of accidents (table 1). Subjects reporting three or more accidents were over twice as likely to report permanent disability (RR $2.16 ; 95 \%$ CI 1.62 to 2.87 ).

Table 2 shows the proportion of accidents presenting for hospital treatment and the proportion resulting in hospital admission. Although only $2 \%$ of accidents presenting for hospital treatment were pedestrian-motor vehicle collisions, $32 \%$ of presentations resulted in hospital admission. In contrast, $30 \%$ of accidents presenting for hospital treatment were work related, but only $6 \%$ required hospital admission.

Table 3 shows the proportion of accidents that resulted in permanent disability by accident type. The proportion of accidents that resulted in permanent disability was substan- tially greater among those requiring hospital admission $(9.8 \%)$ than among those requiring outpatient treatment only $(2.3 \%)$. However, fifty four per cent of permanent disabilities among men, and $74 \%$ among women, resulted from accidents treated in an outpatient setting only. For accidents requiring hospital admission there was no significant gender difference in the proportion resulting in permanent disability. For accidents requiring outpatient treatment the proportion resulting in permanent disability was significantly higher for women $(3.8 \% v 1.9 \%, \mathrm{p}<0.01)$. Disability risk was greatest for pedestrian-motor vehicle collisions, with $6.6 \%$ of collisions resulting in permanent disability. For men, $31 \%$ of permanent disabilities resulted from road traffic accidents, $25 \%$ from work related accidents, and $25 \%$ from sports related accidents. For women, $32 \%$ of permanent disabilities resulted from road traffic accidents, $18 \%$ from work related accidents, and $13 \%$ from accidents in the home.

Fractures constituted $21 \%$ of all injuries reported, but were responsible for $32 \%$ of permanent disabilities. Fractures accounted for half $(7 / 15)$ of the disabling injuries resulting from pedestrian-motor vehicle collisions and one third (34/97) of those resulting from other road vehicle related accidents. Fractures were responsible for $39 \%$ of disabling sports injuries.

Of the 83 permanent disabilities resulting from work related accidents, $82 \%$ involved the hand (19 hand lacerations and nine hand or finger amputations). Of the 34 permanent disabilities resulting from accidents in the home $32 \%$ involved the hand (nine hand lacerations and two hand or finger amputations).

\section{Discussion}

The paucity of information on injury related disability is recognised to be an important barrier to effective preventive action. ${ }^{3}$ The $\mathrm{Na}$ tional Child Development Study represents a unique opportunity to examine the distribution and determinants of injury related disability in a large nationally representative sample of young adults. The results of our analyses have several important implications for the prevention of injury related disability. First, although accidents treated as an outpatient carry a lower risk of permanent disability, because outpatient treated accidents are considerably more common, they account for the majority of injury related permanent disability. This suggests that preventive and therapeutic interventions aimed at the larger number of less severe accidents may have the greatest overall effect on the population burden of disability. Second, while road traffic accidents are a major contributor to both injury mortality and permanent disability, work related accidents, sports related accidents, and accidents in the home also make a substantial contribution to the population burden of disability in this age group. The targeting of preventive efforts towards the major causes of injury mortality will therefore make a smaller impact on population levels of injury related permanent 
disability. Third, the results of these analyses highlight the important contribution of nonlife threatening injuries, in particular hand injuries and limb fractures, to the prevalence of permanent disability in young adults.

There are some methodological issues that may have a bearing on the validity of the results. Among the most important is the potential for bias due to loss to follow up. At age 23 , the follow up rate had declined to $76 \%$ of study participants still alive and resident in Britain. Moreover, certain disadvantaged groups are known to be underrepresented. Because rates of injury are higher among socioeconomically disadvantaged groups, our estimates of the prevalence of injury related disability are likely to be underestimates. ${ }^{7}$

Issues surrounding the definition of disability have been the subject of considerable confusion and controversy in recent years. ${ }^{8}$ In 1980, the World Health Organisation published the International Classification of Impairments, Disabilities and Handicaps. According to this definition disability relates to a restriction or lack of ability in expected human activity resulting from an anatomical, physiological, or psychological impairment. ${ }^{9}$ In the National Child Development Study, permanent disability was self defined. However, in view of the fact that the extent to which an injury results in a restriction of ability is subjective, the use of self report is defensible. The self definition of permanent may be more problematic, particularly in relation to recent injuries. Finally, with a seven year recall period there is the potential for incomplete and inaccurate recall. A New Zealand study found that only $61 \%$ of accident and emergency visits were recalled. ${ }^{10}$ Recall is likely to be influenced by the severity of the event, with less severe injuries being the most likely to be forgotten. If present, recall bias would be expected to result in an overestimation of the risk of injury related disability. Further studies are required that use standardised definitions of disability and shorter recall periods.

In conclusion, the results of our analyses suggest that the targeting of prevention strategies towards the major causes of injury mortality may have a smaller impact on population levels of injury related disability. Nonlifethreatening injuries, in particular injuries to the hand and limb fractures, resulting from accidents in the workplace, the home, and during sports, make a significant contribution to the prevalence of permanent injury related disability in young adults.

This work was funded by the Wolfson Foundation and North West Thames Regional Health Authority (MB) and the West Thames Regional Health Authority (MB) and the Department of Health (CP). We thank the staff at the Social
Statistics Research Unit at City University London for Statistics Research Unit at City
providing access to the NCDS data.

1 Office of Population Censuses and Surveys. Mortality statistics: cause (DH2). London: HMSO, 1992.

2 Department of Health. The health of the nation. London: HMSO, 1991.

3 Department of Health. Fit for the future: second progress report on the health of the nation. London: HMSO, 1995.

4 Yates DW. Scoring systems for trauma. BMF 1990; 301:1090-4.

5 Power C. A review of child health in the 1958 birth cohort: National Child Development Study. Paediatr Perinatal Epidemiol 1992;6:81-110.

6 Power C, Manor O, Fox AJ. Health and class: the early years. London: Chapman Hall, 1991.

7 Office of Population Censuses and Surveys. Occupational mortality: childhood supplement (DS No 8). London: HMSO, 1988

8 Hutchinson T. The classification of disability. Arch Dis Child 1995;73:91-9.

9 World Health Organisation. International classification of impairments, disabilities and handicaps; a manual of classification relating to the consequences of disease. Geneva: WHO, 1980.

10 Chalmers DJ, Cecchi J, Langley JD. Silva PA. Injuries in the 12th and 13th years of life. Aust Paediatr F 1989;25:14-20. 